\title{
Tetraquark-adequate formulation of QCD sum rules
}

\author{
Wolfgang Lucha, ${ }^{1}$ Dmitri Melikhov, ${ }^{1,2,3}$ and Hagop Sazdjian ${ }^{4}$ \\ ${ }^{1}$ Institute for High Energy Physics, Austrian Academy of Sciences, \\ Nikolsdorfergasse 18, A-1050 Vienna, Austria \\ ${ }^{2}$ D. V. Skobeltsyn Institute of Nuclear Physics, M. V. Lomonosov Moscow State University, \\ 119991 Moscow, Russia \\ ${ }^{3}$ Faculty of Physics, University of Vienna, Boltzmanngasse 5, A-1090 Vienna, Austria \\ ${ }^{4}$ Institut de Physique Nucléaire, CNRS-IN2P3, Université Paris-Sud, \\ Université Paris-Saclay, 91406 Orsay, France
}

(Received 13 January 2019; published 10 July 2019)

\begin{abstract}
We study details of QCD sum rules à la Shifman-Vainshtein-Zakharov for exotic tetraquark states. We point out that duality relations for correlators involving exotic currents have fundamental differences compared with the duality relations for the correlators of bilinear quark currents: namely, the $O(1)$ and $O\left(\alpha_{s}\right)$ ( $\alpha_{s}$ the strong coupling constant) terms in the operator product expansion for the exotic correlators exactly cancel against the contributions of the two-meson states on the hadron side of QCD sum rules. As a result, the tetraquark properties turn out to be related to the specific nonfactorizable parts of the exotic Green functions; the relevant nonfactorizable diagrams start at order $O\left(\alpha_{s}^{2}\right)$.
\end{abstract}

DOI: 10.1103/PhysRevD.100.014010

\section{MOTIVATION}

Motivated by increasing experimental evidence for narrow near-threshold hadron resonances with favorable interpretation as tetraquark and pentaquark hadrons (of minimal parton configurations consisting of four and five quarks, respectively) [1-3], extensive theoretical studies of such objects have been carried out. This paper focuses on subtleties of the description of tetraquark mesons by Shifman-Vainshtein-Zakharov (SVZ) sum rules in QCD [4]; we demonstrate that some essential criteria for selecting QCD diagrams relevant for tetraquark properties within QCD sum rules have not been properly taken into account.

For a proper QCD analysis of possible tetraquarks and for the selection of the appropriate Feynman diagrams, the understanding of the four-quark singularities of Feynman diagrams plays a crucial role. In Refs. [5-14], the fourquark singularities of Feynman diagrams describing the four-point functions $\Gamma_{4 j}$ of bilinear quark-antiquark currents $j$ have been carefully studied. References $[13,14]$ introduced the notion of tetraquark-phile ( $T$-phile) diagrams: the $T$-phile diagrams are those Feynman diagrams that have four-quark singularities in the relevant kinematic variable. For $\Gamma_{4 j}$, those diagrams that contain at least two

Published by the American Physical Society under the terms of the Creative Commons Attribution 4.0 International license. Further distribution of this work must maintain attribution to the author(s) and the published article's title, journal citation, and DOI. Funded by SCOAP ${ }^{3}$. gluon exchanges of special topology have been shown to belong to the set of $T$-phile diagrams.

Independently of this line of research, numerous works deal with the analysis of tetraquark states by QCD sum rules (see Refs. [15,16] and references therein). QCD sum rules exploit dispersion representations to calculate QCD Green functions, the time-ordered $(\mathrm{T})$ products of local hadron interpolating currents built of quark and gluon fields, in two different ways: First, one calculates the Green function by converting the T-product into a sum of local operators via Wilson's operator product expansion (OPE). Power corrections, reflecting the modification of quark and gluon propagators at small momentum transfers due to QCD confinement, are given via QCD condensates; they may be calculated, for each QCD diagram, according to wellknown rules [4]. In this way, one obtains the sum rule's theoretical (OPE) side. Second, one calculates the same Green function by inserting a complete set of hadron states. This yields the sum rule's phenomenological (hadronic) side.

For Green functions of currents $j$, the hadron continuum is counterbalanced by the perturbative QCD contributions beyond an appropriate effective threshold. Then, parameters of ordinary hadrons are related to the low-energy region of perturbative QCD diagrams supplemented by appropriate condensate contributions [4]. We demonstrate that, for Green functions involving tetraquark currents, this picture requires serious modifications.

References $[15,16]$ focused on two-point functions $\Pi_{\theta \theta}(x)=\langle\mathrm{T}\{\theta(x) \theta(0)\}\rangle$ of tetraquark interpolating currents $\theta(x)=\bar{q}(x) q(x) \bar{q}(x) q(x)$ and three-point functions $\Gamma_{\theta j j}(0 \mid x, y)=\langle\mathrm{T}\{\theta(0) j(x) j(y)\}\rangle$, involving one tetraquark 
current $\theta$ and two ordinary currents $j(x)=\bar{q}(x) q(x) ;\langle\ldots\rangle$ denotes averaging over the vacuum. (The currents' quark flavor content will be specified below.) All previous applications of SVZ sum rules (SR) to exotic states share one common feature: they adopt the leading-order $O(1)$ diagrams (and sometimes also radiative corrections) and power corrections induced by these diagrams, and borrow exactly the same criteria for continuum subtraction as prescribed for ordinary mesons [4]. Consequently, the tetraquark contribution is found to be dual to the lowenergy spectral integral of the relevant QCD diagrams. Specifically, tetraquarks receive substantial contributions of $O(1)$ and $O\left(\alpha_{s}\right)$ QCD diagrams.

We will prove that the procedures adopted in SR analyses of exotic states $[15,16]$ do not take proper account of the cancellations between the $O(1)$ and $O\left(\alpha_{s}\right)$ diagrams on the OPE side and the two-meson contributions on the hadron side. Let us start with two almost self-evident observations:

(i) QCD sum rules utilize local interpolating currents, so it suffices to consider tetraquark interpolating currents in the form of products of two colorless bilinear quark currents [17]. All other color structures of tetraquark currents are reduced to products of colorless bilinears by Fierz transformations. For the singlet-singlet color structure of $\theta$, any diagram describing $\Pi_{\theta \theta}$ and $\Gamma_{\theta j j}$ may be obtained from the diagrams of $\Gamma_{4 j}$ by merging two pairs of vertices (in the case of $\Pi_{\theta \theta}$ ) or one pair of vertices (in the case of $\left.\Gamma_{\theta j j}\right)$. Technically, the relationship between the Green functions involving tetraquark currents and $\Gamma_{4 j}$ corresponds to defining the local $\theta(x)$ as the product of two point-split colorless currents $j$ by sending their displacement $\delta$ to zero: $\theta(x)=$ $\lim _{\delta \rightarrow 0} j(x) j(x+\delta) .^{1}$

(ii) All previous QCD SR applications to exotic states $[15,16]$ relate tetraquark properties to those contributions to $\Pi_{\theta \theta}$ and $\Gamma_{\theta j j}$ that are obtained by merging vertices in non- $T$-phile diagrams of $\Gamma_{4 j}$. Recall that such contributions to $\Gamma_{4 j}$ have no four-quark cuts [20] and therefore may not be related to tetraquark properties [10-12]. One may therefore doubt that the procedures adopted in Refs. $[15,16]$ are consistent.

We will show that quark-hadron duality relations for Green functions involving exotic tetraquark currents exhibit a specific feature: an exact cancellation of the $O(1)$ and $O\left(\alpha_{s}\right)$ contributions on the OPE side against the two-meson contribution on the hadron side of the SVZ sum rule by virtue of quark-hadron duality relations for correlation functions of colorless currents $j$. (This property is quite general and does not depend on the color structure of

\footnotetext{
${ }^{1}$ The parameter $\delta$ finally sent to zero must not be confused with the finite physical separation between clusters inside tetraquarks discussed in dynamical models for the tetraquark structure, e.g., $[18,19]$.
}

$\theta$ but is most easily demonstrated for $\theta$ taken as the product of two colorless $j$; we therefore present here the analysis of this case.) Hence, upon taking into account these cancellations a QCD SR for any exotic state assumes the following form: the OPE side for $\Pi_{\theta \theta}$ and $\Gamma_{\theta j j}$ has merely contributions of $T$-phile diagrams, obtained from $T$-phile diagrams for $\Gamma_{4 j}$ by merging appropriate vertices; the hadron side has the suspected tetraquark pole and the interacting mesons. One may then assume, similarly to conventional QCD sum rules for ordinary correlators, that the tetraquark contribution is dual to the low-energy part of the $T$-phile contributions to $\Pi_{\theta \theta}$ and $\Gamma_{\theta j j}$.

\section{DIRECT GREEN FUNCTIONS INVOLVING TETRAQUARK CURRENTS}

Let us consider tetraquarks involving two quarks of flavors $a$ and $c$ and two antiquarks of flavors $b$ and $d$, and thus define interpolating currents with two different flavor structures, $\quad \theta_{\bar{a} b \bar{c} d}=j_{\bar{a} b} j_{\bar{c} d}$ and $\theta_{\bar{a} d \bar{c} b}=j_{\bar{a} d} j_{\bar{c} b}$, with $j_{\bar{a} b}=\bar{q}_{a} q_{b}$. We need not specify the Dirac structure of $\theta$, since it does not change our argument.

An appropriate definition of $\theta$ may be given by pointsplitting in the product of two currents $j$. From this perspective, any diagram involving some $\theta$ may be obtained from the four-point function of two currents $j$ studied in detail in [11]. We distinguish between Feynman diagrams where quark flavors in initial and final state are combined in the same way (direct diagrams) and in a different way (recombination diagrams), since they have different topologies and different structures of four-quark singularities. Accordingly, the resulting duality relations should be discussed separately.

\section{A. Two-point function $\Pi_{\theta \theta}^{\mathrm{dir}}$}

Figure 1 shows the direct four-point function $\Gamma_{4 j}^{\mathrm{dir}}$ and the corresponding two-point function of tetraquark currents: only diagrams in (c) are $T$-phile, so the rhs diagrams (a), (b) should drop out from the tetraquark SR. Diagrams with one-gluon exchanges between disconnected loops are null.

To show that this indeed happens, we inspect the OPE and the hadron representation for the two-point direct correlation function $\Pi_{\theta \theta}$, Fig. 2. (In Figs. 2-4, we do not explicitly show power corrections: for any Feynman diagram, they are calculated according to well-known rules [4].) There is an infinite subset of diagrams in the OPE for $\Pi_{\theta \theta}^{\mathrm{dir}}$ that factorize in coordinate space into two parts separated by the red dash-dotted line; the $O(1)$ and $O\left(\alpha_{s}\right)$ diagrams belong to this subset. On the hadron side, there is also an infinite subset of meson contributions that factorize in coordinate space. It is straightforward to check that the OPE factorizable subset exactly equals the hadron factorizable subset by using the QCD SR for the two-point function $\Pi_{j j}$ of ordinary currents $j$ (Fig. 3). Canceling out the equal factorizable parts on both sides of the SR of 


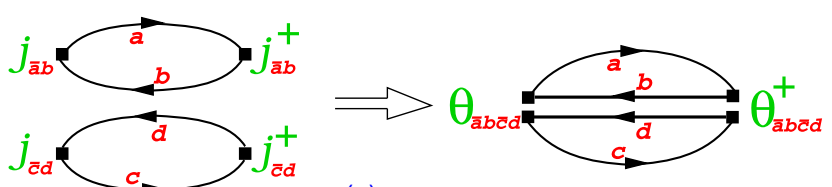

(a)
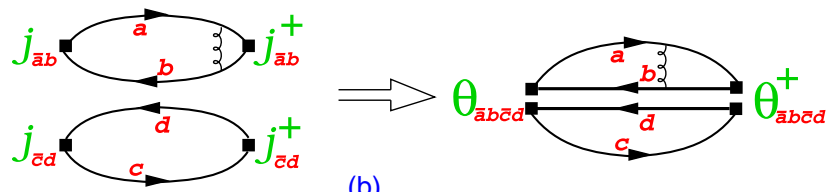

(b)
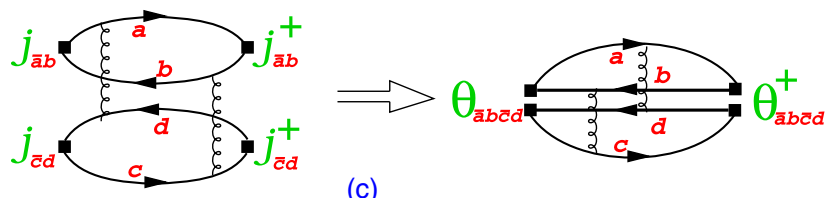

(c)

FIG. 1. Feynman diagrams for a direct two-point function of tetraquark currents $\Pi_{\theta \theta}^{\mathrm{dir}}$, obtained by merging vertices in $\Gamma_{4 j}^{\mathrm{dir}}$. In the left column, diagrams (a) and (b) do not contain four-quark singularities in the $s$ channel, whereas diagram (c) is the lowestorder diagram that contains the four-quark $s$ cut and is thus the only $T$-phile diagram.

Fig. 2, we arrive at the tetraquark SR of Fig. 4. Now, similarly to the case of ordinary mesons, we consider a single spectral representation in $p^{2}$ of the QCD diagrams in the lhs of Fig. 4 and introduce an effective threshold $s_{\text {eff }}$ [21-23] such that the contribution of the QCD diagrams in the lhs of Fig. 4 above $s_{\text {eff }}$ cancels the nonfactorizable meson-meson interaction diagrams on the rhs of Fig. 4. Then, after Borel transformation from $p^{2}$ to Borel variable $\tau$ [4], we obtain the ultimate tetraquark SR

$$
\begin{aligned}
\left(f_{T}^{\bar{a} b \bar{c} d}\right)^{2} \exp \left(-M_{T}^{2} \tau\right)= & \int_{\left(4 m_{q}\right)^{2}}^{s_{\text {eff }}} d s \exp (-s \tau) \rho_{T}^{\operatorname{dir}}(s) \\
& + \text { power corrections. }
\end{aligned}
$$

Here, $4 m_{q} \equiv m_{a}+m_{b}+m_{c}+m_{d}, \quad \rho_{T}^{\text {dir }}$ is the spectral density in the variable $s$ of the rhs of Fig. 1(c) with

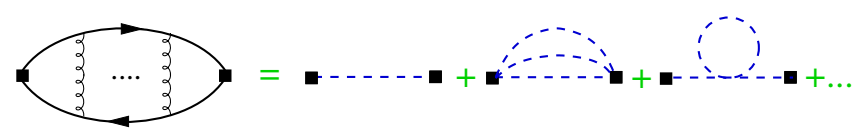

FIG. 3. Conventional QCD SR for the two-point function $\Pi_{j j}^{\text {dir }}$ of ordinary currents $j$ : the lhs shows its OPE (the diagram with two gluon lines with dots in between represents the sum of diagrams with an arbitrary number of gluon exchanges, starting with the quark loop with no gluons); the rhs shows its meson representation.

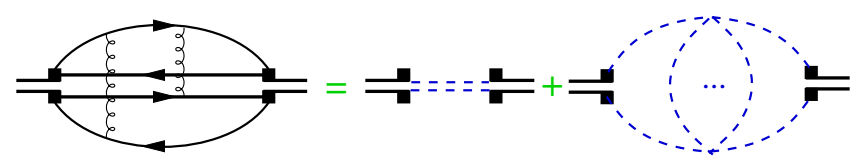

FIG. 4. Final tetraquark QCD SR relating the nonfactorizable OPE contributions to the two-point function $\Pi_{\theta \theta}^{\mathrm{dir}}$ to the sum of the tetraquark pole and the nonfactorizable meson-interaction diagrams. The dots in the meson diagrams denote the sum of meson diagrams of the same (nonfactorizable) topology.

two-gluon exchanges of order $O\left(\alpha_{s}^{2}\right)$. Power corrections in Eq. (2.1) correspond to condensate insertions in the diagram of Fig. 1(c). Power corrections generated by the rhs diagrams in Figs. 1(a) and 1(b) do not contribute to the tetraquark SR (2.1): they cancel against the factorizable meson-meson contributions. $M_{T}$ is the tetraquark mass and $f_{T}^{\bar{a} b \bar{c} d}=\left\langle T\left|\theta_{\bar{a} b \bar{c} d}\right| 0\right\rangle$. Only the $T$-phile diagram of Fig. 1(c) and the corresponding power corrections contribute to the tetraquark SR (2.1).

\section{B. Three-point function $\Gamma_{\theta_{j j}}^{\mathrm{dir}}$}

Direct Green functions $\Gamma_{\theta j j}^{\mathrm{dir}}$ may be found from $\Gamma_{4 j}^{\mathrm{dir}}$ by merging in the latter just one (say, the left) coordinate pair, as shown in Fig. 5; here, only diagram (c) is $T$-phile, so the rhs diagrams (a) and (b) should not contribute to the tetraquark coupling to two mesons. For $\Gamma_{\theta j j}^{\mathrm{dir}}$, this is easily shown: the tetraquark would lead to a pole $1 /\left(p^{2}-M_{T}^{2}\right)$ in $\Gamma_{\theta j j}^{\mathrm{dir}}$ ( $p$ is the total momentum of the currents) with residue

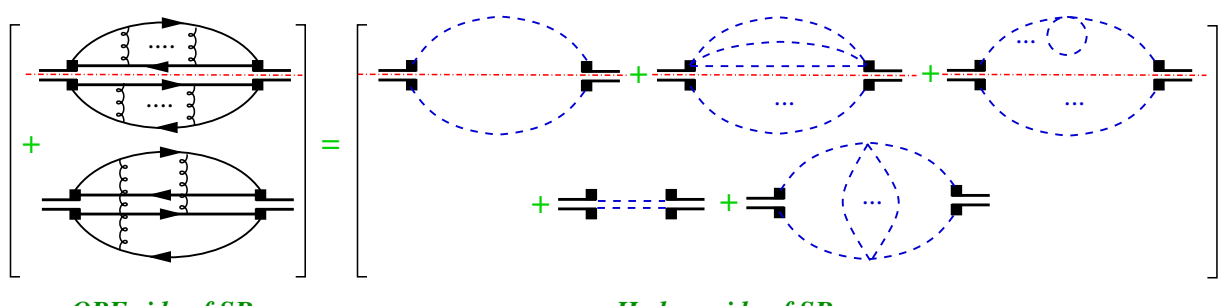

$O P E$ side of $S R$

Hadron side of $S R$

FIG. 2. QCD SR for the two-point function $\Pi_{\theta \theta}^{\mathrm{dir}}$ : The lhs shows its OPE. The rhs shows its meson representation; assuming that the hadron spectrum contains a tetraquark, its contribution appears on the hadron side. The first line on both sides of the SR shows diagrams factorizable into two parts, separated by the red dash-dotted line; the second line on both sides shows nonfactorizable contributions. Note that the set of factorizable diagrams on the OPE side includes diagrams (a) and (b) of the rhs of Fig. 1. Diagrams in the first line on both sides of the SR are equal to each other by virtue of QCD sum rules for $\Pi_{j j}$, Fig. 3 . 

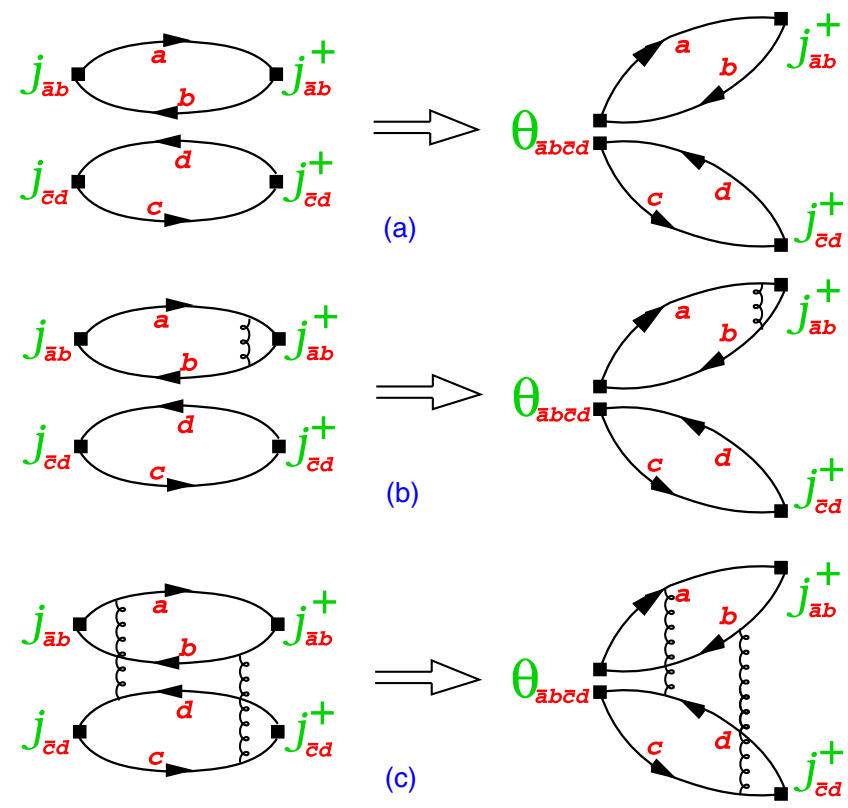

(c)

FIG. 5. Feynman diagrams for a direct three-point function as obtained by merging vertices in $\Gamma_{4 j}^{\mathrm{dir}}$. Diagrams (a), (b) depend on $p^{2}$ at most polynomially and therefore cannot contribute to tetraquark properties. Only diagrams (c) are $T$-phile and therefore contribute to the tetraquark SVZ SR.

related to the tetraquark's coupling to ordinary mesons of appropriate flavor content, $T \rightarrow M_{\bar{a} b} M_{\bar{c} d}$. Clearly, the diagrams of Figs. 5(a) and 5(b) cannot contribute to the pole, as their dependence on $p^{2}$ is at most polynomial, due to traces over quark loops. The Borel transform of the rhs of Figs. 5(a) and 5(b) vanishes. So the rhs diagram of Fig. 5(c) is the lowest-order diagram that gives a nontrivial contribution to the tetraquark pole. Introducing an effective threshold $s_{\text {eff }}$ and performing the Borel transform yields the SR

$$
\begin{aligned}
& f_{T}^{\bar{a} b \bar{c} d} \exp \left(-M_{T}^{2} \tau\right) A\left(T \rightarrow j_{\bar{a} b} j_{\bar{c} d}\right) \\
& \quad=\int_{\left(4 m_{q}\right)^{2}}^{s_{\text {eff }}} d s \exp (-s \tau) \Delta_{T}^{\text {dir }}(s)+\text { power corrections. }
\end{aligned}
$$

Here, $A\left(T \rightarrow j_{\bar{a} b} j_{\bar{c} d}\right)$ is the momentum-space amplitude $\left\langle 0\left|\mathrm{~T}\left\{j_{\bar{a} b}(x) j_{\bar{c} d}(0)\right\}\right| T(p)\right\rangle$ and $\Delta_{T}^{\mathrm{dir}}(s)$ the spectral density in the variable $s$ of the rhs of Fig. 5(c). As before, power corrections generated by non- $T$-phile diagrams do not appear in the tetraquark SR.

\section{RECOMBINATION GREEN FUNCTIONS INVOLVING TETRAQUARK CURRENTS}

For correlators with recombination topology (Fig. 6), where the initial and final quark color singlets have different flavor structures, again only the diagrams of Fig. 6(c) are $T$-phile and contribute to the tetraquark SR. The proof of this is not as straightforward as before.
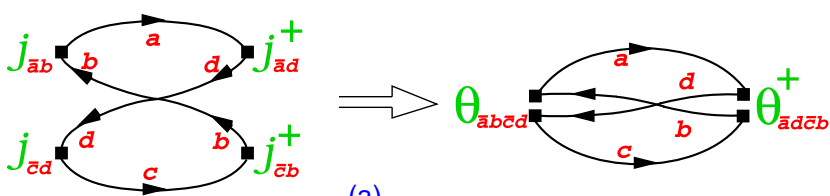

(a)
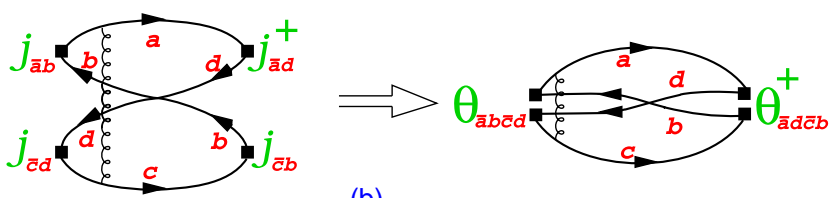

(b)
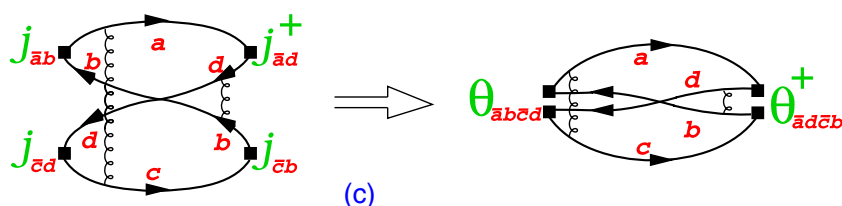

(c)

FIG. 6. Feynman diagrams for a recombination two-point function of tetraquark currents as obtained by merging vertices in the recombination four-point function of bilinear quark currents. Diagrams (a) and (b) on the lhs do not contain fourquark singularities in the $s$ channel. Diagram (c) on the lhs is the lowest-order diagram that contains a four-quark $s$ cut; thus, only diagram (c) on the lhs is the $T$-phile diagram. Also, among the diagrams on the rhs, only diagram (c) contributes to the OPE side of the tetraquark SVZ sum rule (3.1).

However, in Ref. [11] it has been shown that the recombination Green functions (a), (b) on the lhs of Fig. 6 do not contribute to tetraquark poles and are related to meson amplitudes without $s$-channel four-quark singularities. This holds even after merging initial and/or final vertices. Thus, the rhs diagrams (a), (b) of Fig. 6 still do not contribute to the tetraquark pole: only the diagrams in Fig. 6(c) are $T$-phile. Similar considerations apply, mutatis mutandis, to the recombination three-point functions. Hence, we arrive at the tetraquark sum rules

$$
\begin{aligned}
f_{T}^{\bar{a} b \bar{c} d} f_{T}^{\bar{a} d \bar{c} b} \exp \left(-M_{T}^{2} \tau\right)= & \int_{\left(4 m_{q}\right)^{2}}^{s_{\text {eff }}} d s \exp (-s \tau) \rho_{T}^{\mathrm{rec}}(s) \\
& + \text { power corrections }
\end{aligned}
$$

$$
\begin{aligned}
& f_{T}^{\bar{a} b \bar{c} d} A\left(T \rightarrow j_{\bar{a} d} j_{\bar{c} b}\right) \exp \left(-M_{T}^{2} \tau\right) \\
& \quad=\int_{\left(4 m_{q}\right)^{2}}^{s_{\text {eff }}} d s \exp (-s \tau) \Delta_{T}^{\text {rec }}(s)+\text { power corrections. }
\end{aligned}
$$

Here, $4 m_{q} \equiv m_{a}+m_{b}+m_{c}+m_{d}$ and $\rho_{T}^{\mathrm{rec}}(s)$ and $\Delta_{T}^{\mathrm{rec}}(s)$ are the spectral densities in the variable $s$ of the $O\left(\alpha_{s}^{2}\right)$ diagrams with two-gluon exchanges [cf. the rhs of Fig. 6(c)]. The coupling constants are defined by $f_{T}^{\bar{a} b \bar{c} d}=$ $\left\langle T\left|\theta_{\bar{a} b \bar{c} d}\right| 0\right\rangle$ and $f_{T}^{\bar{a} d \bar{c} b}=\left\langle T\left|\theta_{\bar{a} d \bar{c} b}\right| 0\right\rangle$. 


\section{CONCLUSIONS}

We scrutinized the derivation of SVZ sum rules for exotic correlation functions of tetraquark currents $\theta$, namely, two-point functions $\Pi_{\theta \theta}$ and three-point functions $\Gamma_{\theta j j}$ involving one tetraquark current and two bilinear quark currents $j$. Our insights may be summarized as follows:

(i) The duality relations for exotic correlators are fundamentally different from those for correlators of bilinear quark currents: the $O(1)$ and $O\left(\alpha_{s}\right)$ terms in the OPE for exotic correlators exactly cancel against the contributions of two-meson states on the hadron side of QCD SR. Thus, the properly formulated tetraquark SVZ sum rules, Eqs. (2.1), (2.2), (3.1), and (3.2), relate tetraquark properties to specific $T$-phile nonfactorizable parts of the OPE for exotic Green functions; the corresponding nonfactorizable diagrams start at order $O\left(\alpha_{s}^{2}\right)$.

This result is quite general and valid for any color structure of $\theta$, even if of diquark-antidiquark form. Merely the proof of this statement becomes technically more involved: start with the momentum-space diagrams generated by the diquark-antidiquark, perform Fierz transformations reducing the diagrams to the ones of singlet-singlet currents, and only then exploit the ordinary QCD sum rules for the two-point functions of color-singlet currents. Since Fierz transformations do not change a diagram's perturbative order, one again observes the cancellation of the $O(1), O\left(\alpha_{s}\right)$, and the factorizable part of the $O\left(\alpha_{s}^{2}\right)$ contributions on the OPE side against the factorizable two-meson contributions on the hadron side.

(ii) For clarity, we demonstrated the aforementioned general property by considering currents $\theta$ involving quarks of four different flavors, which case exhibits the simplest topology of the direct Green function. If some of the quark flavors in $\theta$ coincide, the direct Green functions receive contributions similar to those of their recombination counterparts. The cancellation of the $O(1), O\left(\alpha_{s}\right)$, and the factorizable part of the $O\left(\alpha_{s}^{2}\right)$ contributions on the OPE side against the factorizable two-meson contributions on the hadron side of QCD sum rules becomes technically more involved but has been verified.

(iii) The cancellation of $O(1), O\left(\alpha_{s}\right)$, and factorizable part of the $O\left(\alpha_{s}^{2}\right)$ contributions in $T$-adequate sum rules holds independently of the color structure of $\theta$. The singlet-singlet color structure, however, has a decisive advantage related to the algorithm of selecting $T$-phile $O\left(\alpha_{s}^{2}\right)$ diagrams: for $\theta$ chosen as the product of two color singlets, the set of $T$-phile diagrams contributing to the properties of some exotic state involves only diagrams obtainable from $T$-phile diagrams of four-point functions of ordinary currents $j$ by merging the appropriate vertices. This observation reduces the analysis of duality relations for tetraquark correlation functions to the analysis of four-quark singularities in four-point functions of currents $j$. For other color structures of $\theta$, the selection criteria for the $T$-phile $O\left(\alpha_{s}^{2}\right)$ diagrams cannot be formulated in such a direct manner: the $T$-phile diagrams for other color structures of $\theta$ are just the Fierz-transformed $T$-phile diagrams established for the singlet-singlet color structure of $\theta$.

The proper application of tetraquark QCD SR requires the knowledge of presently unknown nonfactorizable $O\left(\alpha_{s}^{2}\right)$ radiative corrections and calls for further efforts in order to obtain reliable conclusions about tetraquark candidates.

\section{ACKNOWLEDGMENTS}

The authors are grateful to V. Anisovich, T. Cohen, L. Gladilin, F.-K. Guo, M. Knecht, L. Maiani, B. Moussallam, A. Polosa, V. Riquer, S. Simula, B. Stech, and W. Wang for valuable discussions. D. M. acknowledges support from the Austrian Science Fund (FWF), Project No. P29028. D. M. and H. S. are grateful for support under joint CNRS/RFBR Grant No. PRC Russia/19-52-15022. D. M. has the pleasure of thanking L. Maiani and W. Wang for hospitality during his visit to the T. D. Lee Institute.
[1] A. Esposito, A. Pilloni, and A. D. Polosa, Phys. Rep. 668, 1 (2017).

[2] A. Ali, J. S. Lange, and S. Stone, Prog. Part. Nucl. Phys. 97, 123 (2017).

[3] S. L. Olsen, T. Skwarnicki, and D. Zieminska, Rev. Mod. Phys. 90, 015003 (2018).

[4] M. A. Shifman, A. I. Vainshtein, and V. I. Zakharov, Nucl. Phys. B147, 385 (1979).
[5] S. Coleman, Aspects of Symmetry (Cambridge University Press, Cambridge, United Kingdom, 1985), Chap. 8.

[6] S. Weinberg, Phys. Rev. Lett. 110, 261601 (2013).

[7] M. Knecht and S. Peris, Phys. Rev. D 88, 036016 (2013).

[8] T. D. Cohen and R. F. Lebed, Phys. Rev. D 90, 016001 (2014). 
[9] L. Maiani, A. D. Polosa, and V. Riquer, J. High Energy Phys. 06 (2016) 160.

[10] W. Lucha, D. Melikhov, and H. Sazdjian, Phys. Rev. D 96, 014022 (2017).

[11] W. Lucha, D. Melikhov, and H. Sazdjian, Eur. Phys. J. C 77, 866 (2017).

[12] L. Maiani, A. D. Polosa, and V. Riquer, Phys. Rev. D 98, 054023 (2018).

[13] D. Melikhov, W. Lucha, and H. Sazdjian, Proc. Sci. (EPS-HEP2017) (2017) 390.

[14] W. Lucha, D. Melikhov, and H. Sazdjian, Phys. Rev. D 98, 094011 (2018).

[15] M. Nielsen, F. S. Navarra, and S. H. Lee, Phys. Rep. 497, 41 (2010).
[16] R. M. Albuquerque, J. M. Dias, K. P. Khemchandani, A. Martinez Torres, F. S. Navarra, M. Nielsen, and C. M. Zanetti, arXiv:1812.08207.

[17] R. L. Jaffe, Nucl. Phys. A804, 25 (2008).

[18] S. J. Brodsky, D. S. Hwang, and R. F. Lebed, Phys. Rev. Lett. 113, 112001 (2014).

[19] A. Esposito and A. D. Polosa, Eur. Phys. J. C 78, 782 (2018).

[20] L. D. Landau, Nucl. Phys. 13, 181 (1959).

[21] W. Lucha, D. Melikhov, and S. Simula, Phys. Rev. D 79, 096011 (2009).

[22] W. Lucha, D. Melikhov, and S. Simula, J. Phys. G 37, 035003 (2010).

[23] W. Lucha, D. Melikhov, and S. Simula, Phys. Lett. B 687, 48 (2010). 